\title{
Heat Acclimation of an Adult Female With a Large Surface Area of Grafted Skin
}

\author{
Jonathan E. Wingo, PhD*, David A. Low, PhD*, David M. Keller, PhD, $\dagger$, Scott L. Davis,

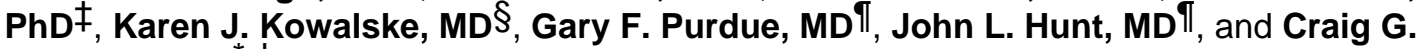 \\ Crandall, $\mathrm{PhD}^{\star}, \dagger$ \\ * Institute for Exercise and Environmental Medicine, Presbyterian Hospital of Dallas, Texas \\ † Department of Internal Medicine, University of Texas Southwestern Medical Center at Dallas, \\ Dallas, Texas \\ ‡ Department of Neurology, University of Texas Southwestern Medical Center at Dallas, Dallas, \\ Texas \\ $\S$ Department of Physical Medicine and Rehabilitation, University of Texas Southwestern Medical \\ Center at Dallas, Dallas, Texas \\ I Department of Surgery, University of Texas Southwestern Medical Center at Dallas, Dallas, Texas
}

\begin{abstract}
Grafted skin has impaired blood flow and sweating responses necessary for heat dissipation. Heat acclimation improves temperature regulation in healthy individuals, but it is unknown whether heat acclimation improves temperature regulation of individuals with large areas of grafted skin. A 33year-old woman with $75 \%$ total body surface area grafted skin 14 years postinjury performed upright cycling exercise at $45 \%$ peak oxygen uptake $(50 \mathrm{~W})$ for seven consecutive days in a climatic chamber set to $40^{\circ} \mathrm{C}$ and $30 \%$ relative humidity. The daily goal was for this patient to exercise 90 minutes (with a 5-minute break at minute 45); however, exercise was stopped when an internal temperature $\left(\mathrm{T}_{\mathrm{c}}\right)$ limit of $39.5^{\circ} \mathrm{C}$ was reached. The $\mathrm{T}_{\mathrm{c}}$ limit was reached during minute 46 of exercise on day 1 of acclimation, but not until minute 65 of exercise on day 7 of acclimation. The increases in $T_{c}$ and heart rate during the first 45 minutes of exercise (the minimum duration completed for all acclimation bouts) were progressively mitigated with successive days of heat acclimation. Sweat sensitivity (the increase in sweat rate per $1{ }^{\circ} \mathrm{C}$ increase in $\mathrm{T}_{\mathrm{c}}$ ) in an area of uninjured skin increased by $\sim 30 \%$ on acclimation day 7 relative to day 1 . Heat acclimation improved thermal tolerance of this patient with a large area of grafted skin, which could increase safety and comfort during thermal stress and/or exercise. (J Burn Care Res 2008;29:848-851)
\end{abstract}

In humans, typical heat loss mechanisms include sweating and increased skin blood flow. ${ }^{1}$ In burn victims, full thickness excision of damaged skin leaves no dermal layer. Because this cutaneous layer houses sweat glands and a vascular network that are both crucial for thermoregulation, heat dissipation, via elevations in skin blood flow and sweating, is impaired. Despite skin grafting over the damaged area, thermoregulatory responses of that skin remain impaired because grafted skin has attenuated reflex cutaneous vasodilation and sweating responses to heat stress. ${ }^{2,3}$ Consequently, individuals with a large proportion of total body surface area (TBSA) of grafted skin may be at an increased risk for heat injury during exercise

Address correspondence to Craig G. Crandall, PhD, Institute for Exercise and Environmental Medicine, Presbyterian Hospital of Dallas, 7232 Greenville Avenue, Suite 435, Dallas, Texas 75231. 
and/or hyperthermic exposure, although the percentage TBSA of grafted or damaged skin necessary to impair whole-body temperature regulation remains unclear.

One way in which individuals with a large percentage TBSA of grafted skin may counter thermal intolerance is via heat acclimation Heat acclimation is the physiological adaptation that occurs on repeated moderate to heavy exercise in a hot environment. ${ }^{4}$ It has been shown to be beneficial in individuals with uninjured skin by lowering cardiovascular and thermal strain during heat exposure after the acclimation regimen. ${ }^{5-7}$ It is unknown, however, whether heat acclimation is effective in improving temperature regulation of individuals with skin grafting over large portions of their body.

\section{CASE DESCRIPTION}

A self-reported recreationally active 33-year-old woman (height $=173 \mathrm{~cm}$; mass $=82.7 \mathrm{~kg}$; body mass index $=27.6 \mathrm{~kg} / \mathrm{m}^{2}, \mathrm{VO}_{2 \text { peak }}=32.4 \mathrm{~mL} \cdot \mathrm{kg}^{-1} \cdot \mathrm{min}^{-1}$ ) with an $88 \%$ TBSA burn ( $75 \%$ TBSA grafted skin) voluntarily consented to undergo a 7-day heat acclimation regimen. Testing on this patient was approved by the Institutional Review Boards at the University of Texas Southwestern Medical Center at Dallas. The patient's injury and subsequent grafting procedures occurred 14 years before this investigation. Before beginning heat acclimation, peak oxygen uptake $\left(\mathrm{VO}_{2 \text { peak }}\right.$; used to identify the exercise intensity for subsequent visits) was measured using an incremental cycle ergometry test to volitional fatigue in a normothermic environment $\left(25^{\circ} \mathrm{C}\right)$. The next seven consecutive visits consisted of the heat acclimation regimen. On each occasion, the patient exercised on a cycle ergometer at $45 \% \mathrm{VO}_{2 \text { peak }}(50$ watts) in a climatic chamber set to $40^{\circ} \mathrm{C}$ and $30 \%$ relative humidity. The daily objective was to exercise for 90 minutes (two 45 -minutes bouts separated by 5 minutes of seated rest). The patient was instructed to consume adequate fluids during the 24 hours between exercise sessions to maintain hydration. Euhydration was confirmed by measuring urine specific gravity before each bout. Additionally, the patient drank $300 \mathrm{~mL}$ of warm (ie, climatic chamber temperature) fluid (Gatorade ${ }^{\mathrm{TM}}$ ) at minute 20 of exercise and again during the break. Heart rate and core temperature $\left(\mathrm{T}_{\mathrm{c}}\right.$; ingestible telemetry pill; HTI Technologies) were measured continuously and ratings of perceived exertion (RPE; Borg scale) ${ }^{8}$ and thermal sensation ${ }^{9}$ were obtained at 10-minutes intervals throughout all acclimation sessions. Whole-body sweat rate and local sweat rate from uninjured and grafted skin were measured on days 1, 4, and 7 . Additionally, carbon monoxide re-breathe derived blood volumes ${ }^{10}$ were measured 1 week before and 1 day after completion of the 7-day heat acclimation regimen.

\section{RESULTS}

The $\mathrm{T}_{\mathrm{c}}$ limit (ie, $39.5^{\circ} \mathrm{C}$ ) was achieved during the 46th minute of exercise on the first day of acclimation (ie, 1 minute into the second exercise period), but not until the 65th minute of exercise on the final day of acclimation (ie, 20 minutes into the second exercise period). Preexercise $\mathrm{T}_{\mathrm{c}}$ for each of these days was within $0.02^{\circ} \mathrm{C}$. Because exercise durations differed across days of the heat acclimation regimen, data are presented for the first 45 minutes of exercise which was the minimum duration achieved for each acclimation day.

The increase in heart rate and $\mathrm{T}_{\mathrm{c}}$ by the 45 th minute of exercise was attenuated over the course of the acclimation regimen (Figure 1). This indicates the acclimation regimen was successful in progressively mitigating cardiovascular and thermal strain. Additionally, sweating sensitivity (ie, the increase in sweat rate per $1{ }^{\circ} \mathrm{C}$ increase in $\mathrm{T}_{\mathrm{c}}$ ) increased in uninjured skin (Figure 2), whereas no measurable sweating was observed in grafted skin regardless of the acclimation day. Improved sweating responses from uinjured skin were not sufficient to increase whole-body sweat rate (data not shown), which was not different over the course of the acclimation regimen. 
In nongrafted individuals, heat acclimation can result in elevations in plasma volume. ${ }^{11-13}$ Consistent with this finding, this patient experienced an approximate $7.5 \%$ increase in blood (4984-5359 mL) and plasma volumes (3199-3439 mL) after heat acclimation.

In addition to the physiological measures described above, it is important to note the decrease in perceptual responses to exercise over the course of the acclimation regimen. RPE at 45 minutes decreased four units from day 1 (18 units) compared with day 7 (14 units) of acclimation. Furthermore, the rating of thermal sensation decreased from 7.5 to 6.5 between acclimation day 1 and day 7 , respectively.

\section{DISCUSSION}

Heat acclimation responses of this patient demonstrate improved thermal tolerance. The difference between $T_{c}$ at the 45th minute of exercise on day 1 vs day 7 was relatively small $\left(0.35^{\circ} \mathrm{C}\right)$, although this may be all that can be achieved in an individual with such a large percentage TBSA of skin with impaired thermoregulation. In nongrafted individuals, acclimation regimens extending to 14 days have been used. ${ }^{14}$ Perhaps a longer acclimation protocol would have elicited even greater responses in this patient, although $\mathrm{T}_{\mathrm{c}}$ and heart rate responses to acclimation typically begin to plateau around day $7.5^{5}$ The attenuation of the increase in $\mathrm{T}_{\mathrm{c}}$ during exercise at the end of the acclimation regimen is comparable in magnitude to that observed by others ${ }^{15,16}$ investigating heat acclimation in women. Furthermore, the change we observed is greater than that seen after the 6th day of an 11-day heat acclimation regimen involving an exercise protocol comparable with that of the current study and using a hotter environment $\left(45^{\circ} \mathrm{C}\right) .{ }^{17}$ On the other hand, some studies have observed a greater attenuation of the increase in $\mathrm{T}_{\mathrm{c}}\left(\sim 0.7^{\circ} \mathrm{C}\right)$ after heat acclimation in nongrafted women. ${ }^{18}$ Differences among studies are likely attributable to highly variable responses to heat acclimation and differences among protocols.

The lack of improvement in whole-body sweat rate in this skin graft patient is inconsistent with observations in nongrafted women. ${ }^{16}$ For instance, Avellini et al ${ }^{16}$ found a 15 to $18 \%$ improvement in sweat rate in women during the preovulatory and postovulatory periods, respectively, after 10 days of treadmill walking for $2 \mathrm{hr} / \mathrm{d}$ in $36^{\circ} \mathrm{C}, 80 \%$ relative humidity. The most likely reason for this difference is the smaller area of non-injured skin of the present subject relative to that in nonburned individuals in the cited study. ${ }^{16}$ Assuming the TBSA of our patient was $1.96 \mathrm{~m}^{2}$ based on the Dubois formula for body surface area, ${ }^{19}$ she would only have approximately $0.23 \mathrm{~m}^{2}$ available for appropriate sweat secretion since sweating is impaired in grafted and scarred skin. ${ }^{2}$

Of particular importance in the present case is the fact that in addition to the physiological changes that occurred in conjunction with heat acclimation (eg, lower heart rate and $\mathrm{T}_{\mathrm{c}}$ at the same time point over the course of acclimation days), this patient perceived the exercise as easier and more comfortable based on lower RPE and thermal sensation ratings at the end of the acclimation regimen. Thus, after heat acclimation, exercise or activity in hot conditions would be better tolerated and therefore may be performed more frequently, which would have positive health benefits for the patient.

Despite improvements in thermal tolerance, this patient remains at a severe disadvantage in terms of an ability to withstand heat stress. The large percentage TBSA of grafted skin, with its inability to appropriately dissipate heat, renders this individual susceptible to development of a heat illness on excessive hyperthermic exposure. Consequently, even with improved thermal tolerance associated with heat acclimation, patients such as this one must still use caution when performing activities in hot conditions. 
An interesting observation from this patient was that her pre-exercise $T_{c}$ averaged $38.1 \pm 0.15^{\circ}$ $\mathrm{C}$, which is higher than the typical $\mathrm{T}_{\mathrm{c}}$ of nonskin grafted individuals. To further investigate this, we tracked $\mathrm{T}_{\mathrm{c}}$ for $\sim 22$ hours between the end of acclimation day 1 and the beginning of acclimation day 2 . This patient's $\mathrm{T}_{\mathrm{c}}$ remained around $38^{\circ} \mathrm{C}$, with the exception of when she went to sleep, at which time her $\mathrm{T}_{\mathrm{c}}$ decreased to approximately $37.1^{\circ} \mathrm{C}$ (Figure 3 ). The patient's baseline oxygen uptake (determined just before the peak oxygen uptake test) was not elevated $(\sim 300-400 \mathrm{~mL} / \mathrm{min})$, so it is unlikely she experienced increased basal metabolic heat production. Instead, a likely rationale for this elevated $\mathrm{T}_{\mathrm{c}}$ response may be that during daily activity heat dissipation is inadequate relative to metabolic heat generation such that $\mathrm{T}_{\mathrm{c}}$ is elevated. On sleeping, metabolism associated with activities of daily living is reduced and, thus, $T_{c}$ is reduced. These responses would occur in the background of typical circadianinduced changes in $T_{\mathfrak{c}}$. Regardless of the mechanism resulting in elevated pre-exercise $T_{\mathfrak{c}}$, this individual, and presumably others with a similar extent of injured skin, begins activity and/or heat stress at an elevated $T_{c}$, so each would have a reduced reserve to increase $T_{c}$ before reaching a critical threshold at which performance suffers and/or heat injury results.

This case study confirms that heat acclimation is successful in improving tolerance to heat stress, even in an individual with $\sim 75 \%$ TBSA of grafted skin, so that activities can be performed with greater safety and less physiological strain. However, sweating remains absent in areas of grafted skin whereas improvements in sweat sensitivity are observed from nongrafted skin. Thus, this individual remains at a high risk for the development of a heat injury because of compromised heat dissipation capability. These findings raise the question of whether similar, or perhaps greater, improvements in heat acclimation responses would occur in individuals with a lower percentage TBSA of grafted skin.

\section{Acknowledgments}

This study was supported by a grant GM68865 from National Institute of General Medical Sciences (NIGMS) (to C.G.C.).

We thank Amanda Fralin, RN for her assistance with this project.

\section{References}

1. Sawka, MN.; Wenger, CB. Physiological responses to acute exercise-heat stress. In: Pandolf, KB.; Sawka, MN.; Gonzalez, RR., editors. Human performance physiology and environmental medicine at terrestrial extremes. Indianapolis, IN: Benchmark; 1988. p. 97-151.

2. Davis SL, Shibasaki M, Low DA, et al. Impaired cutaneous vasodilation and sweating in grafted skin during whole-body heating. J Burn Care Res 2007;28:427-34. [PubMed: 17438492]

3. Davis SL, Shibasaki M, Low DA, et al. Skin grafting impairs postsynaptic cutaneous vasodilator and sweating responses. J Burn Care Res 2007;28:435-41. [PubMed: 17438491]

4. Wenger, CB. Human heat acclimatization. In: Pandolf, KB.; Sawka, MN.; Gonzalez, RR., editors. Human performance physiology and environmental medicine at terrestrial extremes. Indianapolis, IN: Benchmark; 1988. p. 153-97.

5. Eichna LW, Park CR, Nelson N, Horvath SM, Palmes ED. Thermal regulation during acclimatization in a hot, dry (desert type) environment. Am J Physiol 1950;163:585-97. [PubMed: 14799635]

6. Roberts MF, Wenger CB, Stolwijk JA, Nadel ER. Skin blood flow and sweating changes following exercise training and heat acclimation. J Appl Physiol 1977;43:133-7. [PubMed: 893254]

7. Piwonka RW, Robinson S. Acclimatization of highly trained men to work in severe heat. J Appl Physiol 1967;22:9-12. [PubMed: 6017659]

8. Borg GA, Noble BJ. Perceived exertion. Exerc Sport Sci Rev 1974;2:131-53. [PubMed: 4466663]

9. Toner MM, Drolet LL, Pandolf KB. Perceptual and physiological responses during exercise in cool and cold water. Percept Mot Skills 1986;62:211-8. [PubMed: 3960662] 
10. Burge CM, Skinner SL. Determination of hemoglobin mass and blood volume with CO: evaluation and application of a method. 1995;79:623-31.

11. Wyndham CH, Benade AJ, Williams CG, Strydom NB, Goldin A, Heyns AJ. Changes in central circulation and body fluid spaces during acclimatization to heat. J Appl Physiol 1968;25:586-93. [PubMed: 5687365]

12. Harrison MH, Edwards RJ, Graveney MJ, Cochrane LA, Davies JA. Blood volume and plasma protein responses to heat acclimatization in humans. J Appl Physiol 1981;50:597-604. [PubMed: 7251450]

13. Senay LC, Mitchell D, Wyndham CH. Acclimatization in a hot, humid environment: body fluid adjustments. J Appl Physiol 1976;40:786-96. [PubMed: 931907]

14. Armstrong LE, Maresh CM. The induction and decay of heat acclimatisation in trained athletes. Sports Med 1991;12:302-12. [PubMed: 1763248]

15. Weinman KP, Slabochova Z, Bernauer EM, Morimoto T, Sargent F. Reactions of men and women to repeated exposure to humid heat. J Appl Physiol 1967;22:533-8. [PubMed: 6020239]

16. Avellini BA, Kamon E, Krajewski JT. Physiological responses of physically fit men and women to acclimation to humid heat. J Appl Physiol 1980;49:254-61. [PubMed: 7400008]

17. Horstman DH, Christensen E. Acclimatization to dry heat: active men vs. active women. J Appl Physiol 1982;52:825-31. [PubMed: 7085415]

18. Fein JT, Haymes EM, Buskirk ER. Effects of daily and intermittent exposures on heat acclimation of women. Int J Biometeorol 1975;19:41-52. [PubMed: 1193767]

19. Gagge, AP.; Gonzalez, RR. Mechanisms of heat exchange: biophysics and physiology. In: Fregly, MJ.; Blatteis, CM., editors. Handbook of physiology, section 4: environmental physiology. New York: Oxford University Press; 1996. p. 45-84. 

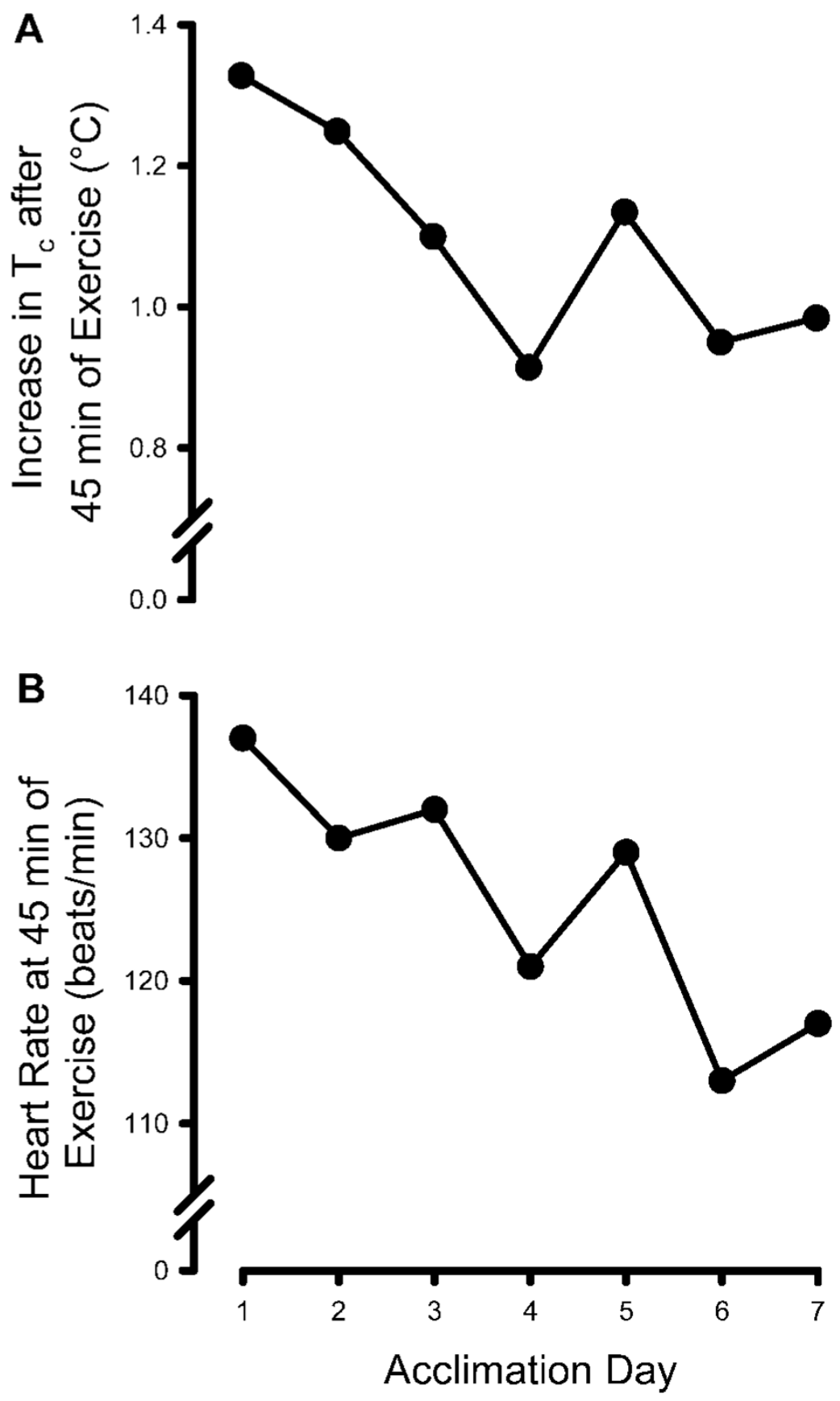

Figure 1.

A. Increase in core temperature $\left(T_{c}\right)$ during the first 45 minutes of exercise across acclimation days. B. Average heart rate during minute 45 of exercise across acclimation days. 


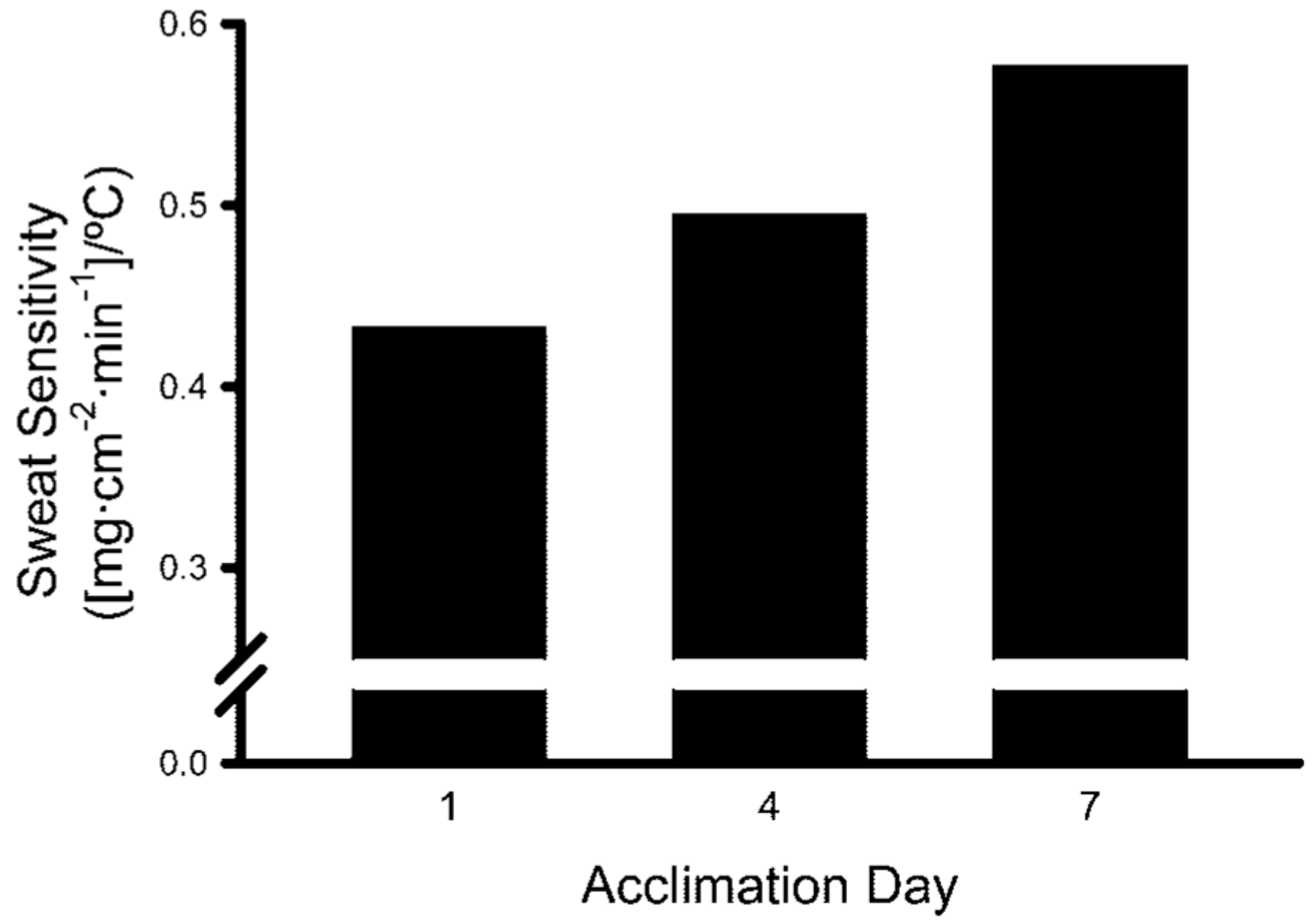

Figure 2.

Sweat sensitivity, defined as the increase in sweat rate per $1{ }^{\circ} \mathrm{C}$ increase in $\mathrm{T}_{\mathrm{c}}$, from non-grafted skin on days 1, 4, and 7 of acclimation. Units of sweat sensitivity are milligrams of sweat per square centimeter of skin surface area per minute relative to internal temperature $([\mathrm{mg}$. $\left.\left.\mathrm{cm}^{-2} \cdot \min ^{-1}\right] /{ }^{\circ} \mathrm{C}\right)$. 


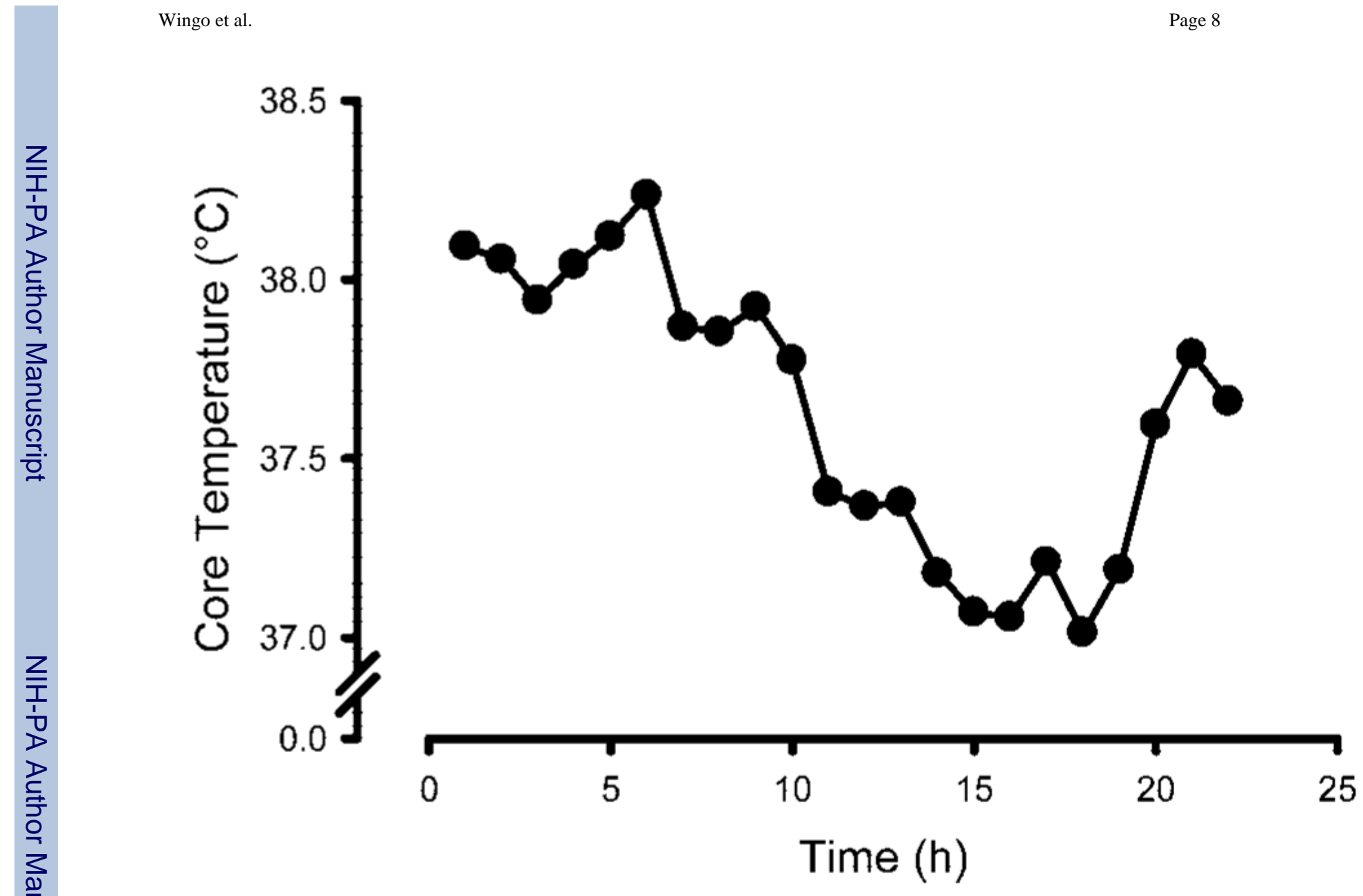

Figure 3.

Continuous core temperature tracking for $\sim 22$ hours during the period between the first and second acclimation bouts. Notice the large decrease in core temperature around the time the patient slept. 